\title{
Expression of MUC1 (EMA) and E-cadherin in renal cell carcinoma: a systematic immunohistochemical analysis of 188 cases
}

\author{
Cord Langner ${ }^{1}$, Manfred Ratschek ${ }^{1}$, Peter Rehak ${ }^{2}$, Luigi Schips ${ }^{3}$ and Richard Zigeuner ${ }^{3}$ \\ ${ }^{1}$ Institute of Pathology; ${ }^{2}$ Department of Surgery, Division of Biomedical Engineering \& Computing and \\ ${ }^{3}$ Department of Urology, University of Graz, Medical School, Graz, Austria
}

\begin{abstract}
MUC1 (epithelial membrane antigen) is a membrane-associated mucin known to interfere with both cell-cell and cell-matrix adhesions. Overexpression has been associated with poor prognosis in a variety of cancers. We investigated the expression of MUC1 (using two different antibodies, MA695 and E29) and E-cadherin in renal cell carcinomas (137 conventional, 23 chromophobe, 20 papillary, and eight unclassified tumors) with respect to diagnostic and prognostic significance using a tissue microarray technique. Immunoreactivity was correlated with histological subtype, pT-stage, and grade using the $\chi^{2}$ test or the Fisher's exact test, respectively. Impact on disease-free survival was analyzed using the Kaplan-Meier method and the log-rank test. Immunoreactivity of more than $10 \%$ of cancer cells with MA695, E 29, and E-cadherin antibodies was found in 112/133 (84\%), $86 / 133(65 \%)$, and $7 / 131(5 \%)$ conventional, $20 / 22(91 \%), 19 / 22(86 \%)$, and $21 / 22(95 \%)$ chromophobe, $13 / 20$ $(65 \%), 8 / 20(40 \%)$, and $3 / 20(15 \%)$ papillary as well as $5 / 8(63 \%), 5 / 8(63 \%)$, and $4 / 8(50 \%)$ unclassified carcinomas, respectively. The two different MUC1 antibodies yielded comparable staining results. A diffuse cytoplasmic staining pattern for MUC1 was found exclusively in chromophobe carcinomas, whereas conventional and papillary subtypes showed predominantly membranous staining $(P<0.0001)$. Regarding papillary carcinomas, MUC1 was predominantly associated with type $1(P=0.0001)$, and E-cadherin with type 2 $(P=0.049)$ tumors. The cellular staining pattern of MUC1 in conventional tumors was related to $\mathrm{pT}$-stage $(P=0.002)$ and tumor grade $(P=0.001)$ : Low-stage (pT1/pT2) and grade (G1/G2) tumors showed a predominantly apical membranous staining, high-stage (pT3a/pT3b) and grade (G3/G4) tumors a predominantly circumferential membranous staining (with or without additional diffuse cytoplasmic immunoreactivity), which, in the conventional subtype, was associated with poor prognosis $(P<0.0001)$. In conclusion, MUC1 and E-cadherin are diagnostically and prognostically useful markers in renal tumor pathology, especially when cellular staining patterns are considered.
\end{abstract}

Modern Pathology (2004) 17, 180-188, advance online publication, 5 December 2003; doi:10.1038/modpathol.3800032

Keywords: kidney; cancer; MUC1; EMA; E-cadherin; differential diagnosis; prognosis

Mucins are high-molecular-weight ( $>200 \mathrm{kDa})$ glycoproteins with oligosaccharides attached to an apomucin protein backbone (core peptide) by Oglycosidic linkage. ${ }^{1,2}$ They are mainly synthesized by epithelial cells and can be classified as secretory (gel-forming) and membrane-associated forms. MUC1 is a transmembrane protein with a large extracellular tandem repeat domain and can be found on the apical surface of almost all glandular and ductal epithelial cells. In the kidney, MUC1 expression was noted on the luminal surfaces of

Correspondence: Dr C Langner, Department of Pathology, University of Graz Medical School, Auenbruggerplatz 25, A-8036 Graz, Austria.

E-mail: cord.langner@uni-graz.at

Received 17 June 2003; revised 10 September 2003; accepted 14 October 2003; published online 5 December 2003 distal tubule and collecting duct epithelium. ${ }^{3-6}$ MUC1 is also known as CD227 or epithelial membrane antigen (EMA). Although EMA is well known in diagnostic histopathology, we prefer the term MUC1 in this study, as it reflects the biological properties and functions of the molecule and furthermore allows its categorization into the growing group of mucin glycoproteins (MUC1-16).

The extracellular domain, which forms an elongated structure protruding from the cell surface, is responsible for the protective function of MUC1 and is implicated in immunoregulation. ${ }^{7}$ Furthermore, the length, rigidity, and negative charge of the extracellular tandem repeat domain of MUC1 is believed to interfere with both cell-cell and cellmatrix adhesions by masking smaller membraneassociated molecules through steric hindrance and to keep ducts and gland lumina open. ${ }^{8,9}$ On the other 
hand, the intracellular domain of MUC1 is implicated in transmembrane signal transduction through interaction with the epidermal growth factor receptor and activation of mitogenic MAP kinase pathways. ${ }^{10}$ Finally, the intracellular domain was shown to bind beta-catenin leading to inhibition of E-cadherin-mediated cell-cell adhesion. ${ }^{11-13}$

Aberrant de novo expression or overexpression of MUC1 was demonstrated in several human malignancies. Tumor cells often show a loss of polarity and a switch from apical to circumferential staining. The covering of the entire cell membrane with MUC1 is supposed to play an important role during invasion and metastasis due to reduced cell adhesion. ${ }^{14}$ Compared to non-neoplastic tissue, however, tumor-associated MUC molecules are commonly underglycosylated, which may lead to a demasking of the peptide antigens facilitating their immunohistochemical detection. ${ }^{15,16}$ Nevertheless, over expression of MUC1 in cancer tissues has been associated with poor prognosis in gastrointestinal adenocarcinomas, ${ }^{15,17-22}$ esophageal squamous cell carcinomas, ${ }^{23}$ endometrial adenocarcinomas, ${ }^{24}$ and non-small-cell lung cancers. ${ }^{25}$ In breast cancer, the situation seems to be more complex. In most studies, increased MUC1 expression was found in lowgrade, estrogen-receptor-positive tumors, and was associated with better prognosis. Regarding staining pattern, however, high cytoplasmic immunoreacti vity was associated with worse prognosis. ${ }^{26}$ Regarding renal cell carcinomas, MUC1 expression is common in conventional tumors and has been reported to be associated with tumor grade and stage. ${ }^{3-6,27,28}$ Prognostic relevance, however, is still under debate, since some authors noticed a significant influence on patients' outcome, ${ }^{3-5,27}$ whereas others failed to detect an influence on tumor aggressiveness in experimental systems. ${ }^{29}$

The cadherins constitute a family of transmembrane glycoproteins that function as calcium-dependent homotypic adhesion molecules and are expressed by most epithelia. Currently, more than 20 different tissue-specific cadherins are known. ${ }^{30}$ Decreased E-cadherin expression correlates with tumor aggressiveness in most carcinomas. In renal cancer, however, E-cadherin expression is infrequently seen and association with outcome is not well established. ${ }^{31-36}$

The current study was designed to investigate the expression of MUC1 applying two different monoclonal antibodies with respect to its diagnostic and prognostic impact and association with E-cadherin immunoreactivity in a large number of renal cell carcinomas including all histological subtypes.

\section{Material and methods}

\section{Case Selection}

A total of 188 paraffin-embedded specimens of renal cell carcinomas from patients (112 males, 76 females) who underwent radical nephrectomy between 01/1995 and 06/2002 and did not receive adjuvant therapy were retrieved from the files of our department for further analysis. The mean age of patients at operation was 62 years (range 28-85). All specimens were re-evaluated with respect to tumor stage, grade, and histological subtypes by two pathologists (CL and MR). Tumor stages were assessed according to the UICC 2002 issue of the TNM-system, ${ }^{37}$ and nuclear grading was based on the Fuhrman grading system. ${ }^{38}$ Histological subtypes were assessed according to the consensus classification of renal cell neoplasia: ${ }^{39,40}$ The mate rial studied comprised conventional or clear cell ( $n=137,73 \%$; including nine tumors with small foci of sarcomatoid change and four tumors with predominant sarcomatoid morphology showing only small residual foci of conventional tumor), papillary $(n=20,11 \%$; including 12 type 1 and eight type 2 tumors) and chromophobe $(n=23,12 \%)$ carcinomas. Eight tumors (4\%) did not fit into either category and were included as renal cell carcinoma, unclassified. Details regarding pT-stages and grades related to histological subtypes are listed in Table 1. Two specimens of non-neoplastic renal tissue were analyzed for comparison.

Table 1 Tumor stage and grade related to histological subtypes of renal cell carcinoma

\begin{tabular}{|c|c|c|c|c|c|c|c|c|}
\hline \multirow[t]{2}{*}{ Stage/grade } & \multicolumn{2}{|c|}{ Conventional $\mathrm{N}=137$} & \multicolumn{2}{|c|}{ Chromophobe $\mathrm{N}=23$} & \multicolumn{2}{|c|}{ Papillary $\mathrm{N}=20$} & \multicolumn{2}{|c|}{ Unclassified $\mathrm{N}=8$} \\
\hline & $\mathrm{N}$ & $\%$ & $\mathrm{~N}$ & $\%$ & $\mathrm{~N}$ & $\%$ & $\mathrm{~N}$ & $\%$ \\
\hline PT1a & 50 & 36 & 6 & 26 & 7 & 35 & 3 & 38 \\
\hline PT1b & 21 & 15 & 3 & 13 & 4 & 20 & 1 & 13 \\
\hline PT2 & 5 & 4 & 3 & 13 & 3 & 15 & 2 & 25 \\
\hline РT3a & 27 & 20 & 6 & 26 & 4 & 20 & 2 & 25 \\
\hline РT3b & 34 & 25 & 5 & 22 & 2 & 10 & 0 & 0 \\
\hline G1 & 18 & 13 & 0 & 0 & 1 & 5 & 0 & 0 \\
\hline G2 & 71 & 52 & 14 & 61 & 13 & 65 & 3 & 38 \\
\hline G3 & 44 & 32 & 9 & 39 & 6 & 30 & 5 & 63 \\
\hline G4 & 4 & 3 & 0 & 0 & 0 & 0 & 0 & 0 \\
\hline
\end{tabular}




\section{Immunohistochemistry}

For immunohistochemical evaluation, a tissue microarray (TMA) technique was used that allows staining of a large number of specimens on one slide. TMAs were constructed using a manual tissue arraying instrument (Beecher, Silver Spring, MD, USA). The details of this technique have been described previously. ${ }^{41-43}$ Three cylindrical core biopsies, $0.6 \mathrm{~mm}$ in diameter, were taken from different sites of each tumor and arrayed in a recipient paraffin TMA block. For the detection of MUC1, two different mouse monoclonal antibodies were used: MA695 (NCL-MUC-1, Novocastra Laboratories Ltd, Newcastle upon Tyne, UK), which recognizes a carbohydrate epitope of the human MUC1 glycoprotein, and E29 (EMA, DAKO, Glostrup, Denmark), which binds to MUC1 independent of its glycosylation. ${ }^{44}$ For the detection of E-cadherin, the mouse monoclonal antibody 4A2C7 (Zymed Laboratories Inc., San Francisco, USA) was used. Sections $(4 \mu \mathrm{m})$ were mounted on Superfrost ${ }^{\mathrm{m}}$ slides for immunohistochemical staining using automated immunostainers (for MA695 and E-cadherin DAKOAutostainer, Universal Staining System; for E29 VENTANA ES, Ventana, Strasbourg, France). Briefly, TMA sections were deparaffinized, rehydrated in graded alcohols, and treated for $5 \mathrm{~min}$ with $1 \%$ $\mathrm{H}_{2} \mathrm{O}_{2}$. The sections were subjected to antigen retrieval (for MA695 and E-cadherin, Epitope Retrieval Solution, DAKO, Code No. K 5205, for $40 \mathrm{~min}$ at $98^{\circ} \mathrm{C}$; for E29, Protease 1 Reagent, Ventana, for $8 \mathrm{~min}$ at room temperature) and subsequently incubated for $30 \mathrm{~min}$ with the primary antibodies (MA695 1:100, E29 1:500, E-cadherin ready to use). The binding of the primary antibodies was assessed by the DAKO ChemMate ${ }^{\text {mw }}$ detection kit (for MA695), the DAKO LSAB ${ }^{\mathbb{}} 2$ System HRP (AEC) detection kit (for E-cadherin), and the Ventana Basic DAB detection kit (for E29), respectively.

\section{Immunohistochemical Evaluation and Controls}

Immunoreactivity was independently evaluated semiquantitatively by two pathologists (CL and MR) who were blinded to the clinicopathologic data, especially pT-stage and patients' outcome. Discrepancies were resolved by re-examination of the slides by both investigators using a doubleheaded microscope. Immunoreactivity was catego rized as follows: 0 , no reactivity; 1 , less than $10 \%$ of cancer cells positive; $2,10-25 \%$ positive; $3,25-50 \%$ positive; $4,50-75 \%$ positive; $5,75-90 \%$ positive; and 6 , more than $90 \%$ of cancer cells positive. For comparative analysis, specimens showing positive immunostaining in more than $10 \%$ of tumor cells were considered positive in accordance with previous studies. ${ }^{4,27}$ In addition to the semiquantitative evaluation, staining patterns for MUC1 were classified as either (predominantly) membranous or (predominantly) diffuse. In a further step, the membranous pattern was further subdivided into a predominantly apical or a predominantly circumferential immunoreactivity (with or without an additional diffuse cytoplasmic immunoreactivity). Non-neoplastic breast tissue served as positive control for all three antibodies and was included in each run. Negative controls included omission of the primary antibody and incubation with DAKO ChemMate $^{\mathrm{TM}}$ Antibody Diluent (Code No. S 2022).

\section{Statistical Analysis}

Subgroups according to pT-stage, grading, and histological subtypes were compared with respect to differences in immunoreactivity using the $\chi^{2}$ test or the Fisher's exact test, respectively. The relationship between MUC1 and E-cadherin was also tested by the $\chi^{2}$ test, while the immunoreactivities of the two different MUC1 antibodies were compared using the weighted $\kappa$ correlation coefficient. Regarding prognosis, only the conventional subtype was analyzed since the sample sizes of the other histotypes were too small to allow a separate analysis. To assess the representativeness of the data set, a Cox proportional hazards regression model for tumor stage and grade was performed. Disease-free survival was investigated using the Kaplan-Meier method and compared by the logrank test analyzing different cutoff levels of immunoreactivity to detect the cutoff with the highest impact on prognosis. Since the results of the statistical tests are interpreted exploratorily, no corrections were made for multiple testing.

\section{Results}

\section{Non-neoplastic Renal Tissue}

In normal renal tissue, a strong and diffuse cytoplasmic expression of MUC1 with apical polarity was seen with both antibodies in the distal tubule and collecting duct epithelium. Proximal tubules lacked MUC1 immunoreactivity (Figure 1a). E-cadherin was strongly expressed in the cell membranes of distal tubule and (to a minor extent) collecting duct epithelium, whereas the cytoplasm lacked immunoreactivity. In proximal tubule epithelium, E-cadherin immunoreactivity was faint, only focal, and confined to the basal parts of the cytoplasm, whereas the cell membranes remained unstained (Figure 3a).

\section{Renal Cell Carcinoma}

Cancer tissue allowing a reliable evaluation of immunoreactivity for MUC1 was present in 183/188 $(97 \%)$ cases, and sufficient tissue for evaluation of E-cadherin immunoreactivity was present in 181/188 (96\%) cases. The results of immunohistochemical 

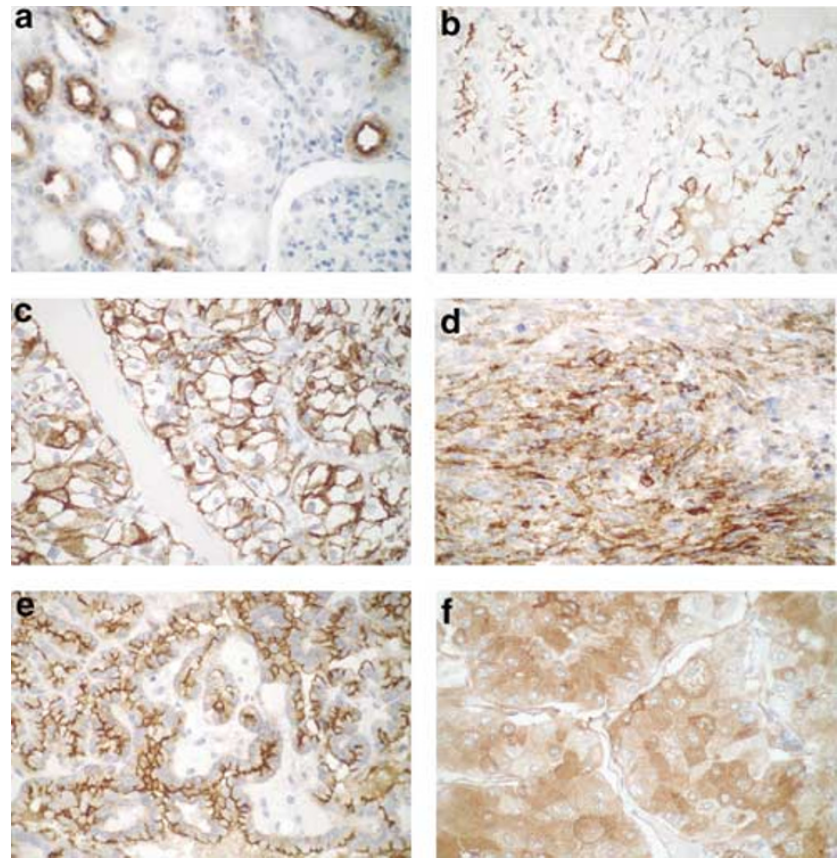

Figure 1 MUC1 immunoreactivity in non-neoplastic renal tissue and renal cell carcinoma. (a) Staining of normal distal tubule epithelium with apical membranous accentuation. Apical (b) and circumferential (c) membranous staining in moderately differentiated conventional carcinoma. Additional diffuse cytoplasmic staining (d) in sarcomatoid areas of poorly differentiated conventional-type cancer. Strong apical membranous MUC1 expression in papillary (e), characteristic diffuse cytoplasmic staining pattern of chromophobe tumors (f).

staining related to histological subtypes, tumor stages, and grades are summarized in Table 2. Like in non-neoplastic tubule epithelium, both MUC1 antibodies showed identical staining patterns (Figure 2a, b). E29 immunoreactivity, however, was slightly weaker compared to MA695. Nevertheless, a statistically significant correspondence of the two MUC1 antibodies (MA695 and E29) was found regarding both qualitative (positive or negative) and semiquantitative staining results $\left(P<0.0001 ; \chi^{2}\right.$ test; weighted $\kappa=0.67 ; 95 \%$ confidence inter$\mathrm{val}=0.58-0.76)$. The overall accuracy between the two antibodies was $86 \%$ with each $9 \%$ negative and $77 \%$ positive staining. E-cadherin showed no significant association with MUC1 (weighted $\kappa=0.008 ; 95 \%$ confidence interval $=-0.08-0.06$; $P=0.8)$. Regarding semiquantitative analysis, MUC1 (MA695) immunoreactivity of more than $50 \%$ of tumor cells was found in 68/133 (51\%) conventional, 15/22 (68\%) chromophobe, and 11/20 $(55 \%)$ papillary cancers.

For MUC1 and E-cadherin, no association with pT-stage was found among the histological subtypes. Regarding tumor grade, papillary carcinomas showed stronger MUC1 immunoreactivity in lowgrade (MA695: G1/G2 12/14 (86\%) vs G3 1/6 (17\%); $P=0.007$, Fisher's exact test; E29: G1/G2 8/14 (57\%) vs G3 0/6 (0\%); $P=0.04$, Fisher's exact test) tumors, whereas for the other subtypes no association was

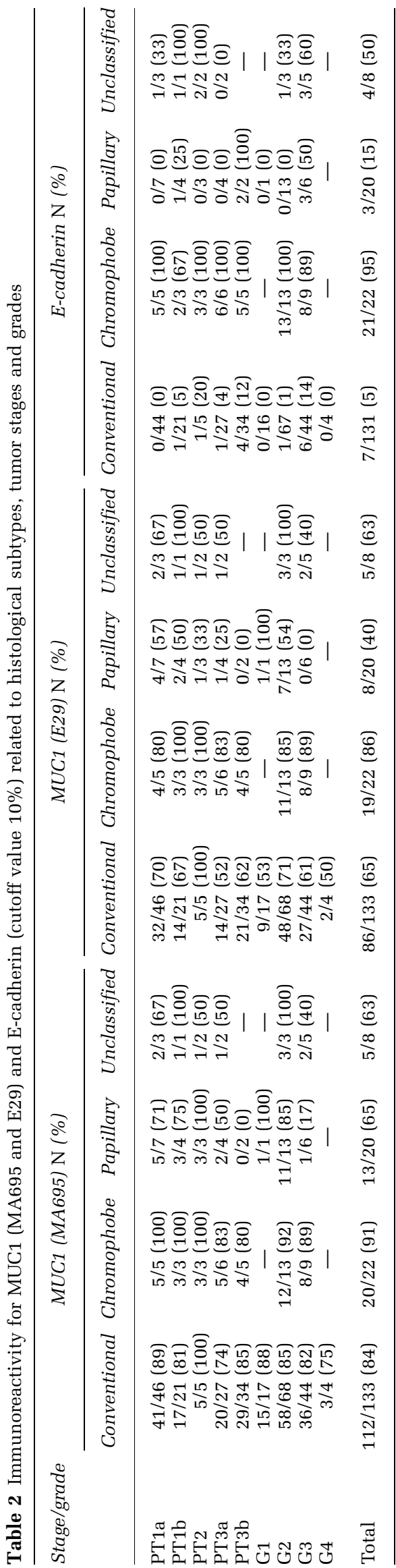



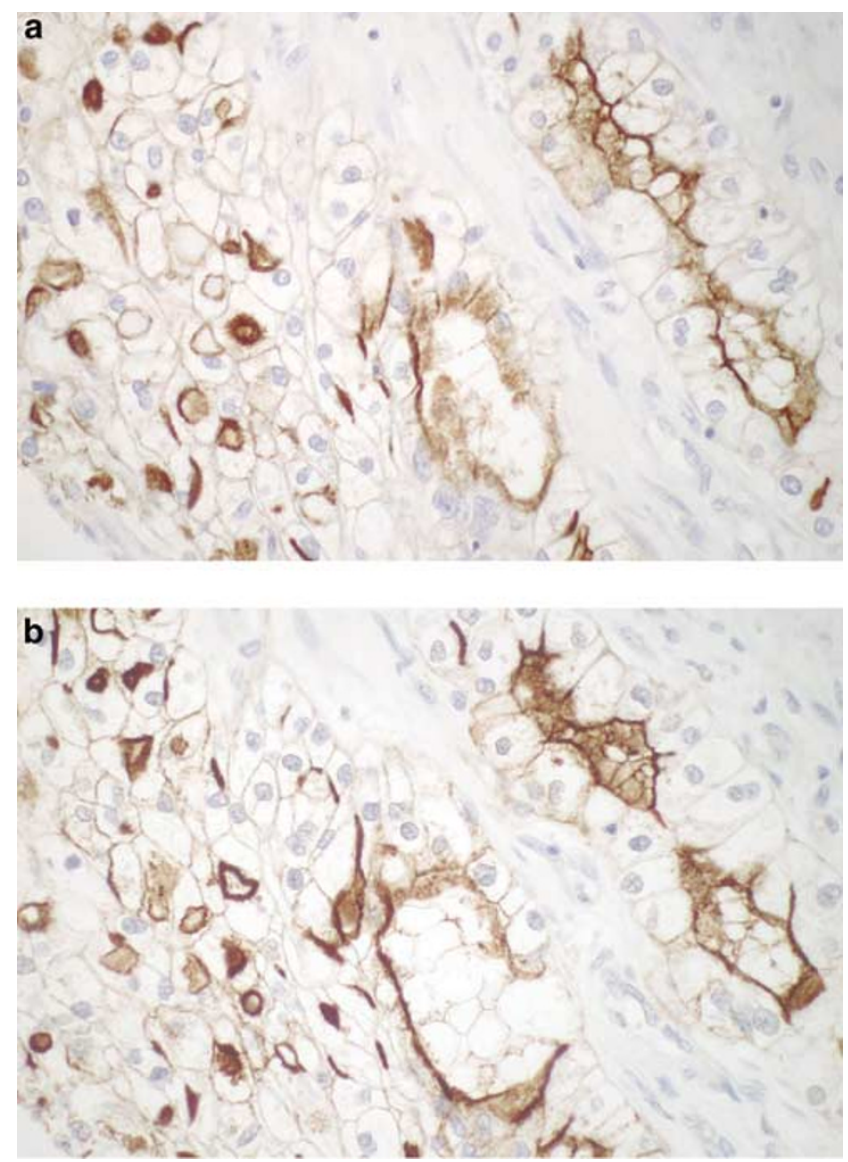

Figure 2 Conventional renal cell carcinoma showing identical staining patterns with the two different MUC1 antibodies MA695 (a) and E29 (b) in serial sections.

found. E-cadherin immunoreactivity, however, was seen more often in high-grade conventional (G1/G2 1/83 (1\%) vs G3/G4 6/48 (13\%); $P=0.01$, Fisher's exact test) and high-grade papillary (G1/G2 0/14 (0\%) vs G3/G4 3/6 (50\%); $P=0.02$, Fisher's exact test) renal cancers.

Regarding histological subtypes, statistically significant associations were found for MA695 $\left(P=0.03 ; \chi^{2}\right.$ test $)$ E29 $\left(P=0.01 ; \chi^{2}\right.$ test $)$, and E-cadherin $\left(P<0.0001 ; \chi^{2}\right.$ test). E-cadherin immunoreactivity showed a statistically significant difference, especially between conventional $(7 / 131,5 \%)$ and chromophobe carcinomas (21/22, 95\%; $P<0.0001$, Fisher's exact test; Figure 3b). Moreover, E-cadherin immunoreactivity of more than $50 \%$ of cancer cells was found in 15/22 (68\%) chromophobe compared to $1 / 131$ (1\%) conventional carcinomas $(P<0.0001$, Fisher's exact test). Regarding papillary carcinomas, MA695 immunoreactivity was found in $12 / 12(100 \%)$ type 1 and $1 / 8(13 \%)$ type 2 papillary carcinomas $(P=0.0001$, Fisher's exact test; Figure 1e), E29 immunoreactivity in 8/12 (67\%) type 1 and $0 / 8(0 \%)$ type 2 papillary carcinomas $(P=0.005$, Fisher's exact test), and E-cadherin in none of 12 type 1 compared to $3 / 8(38 \%)$ type 2 papillary carcinomas $(P=0.049$, Fisher's exact test), respec-
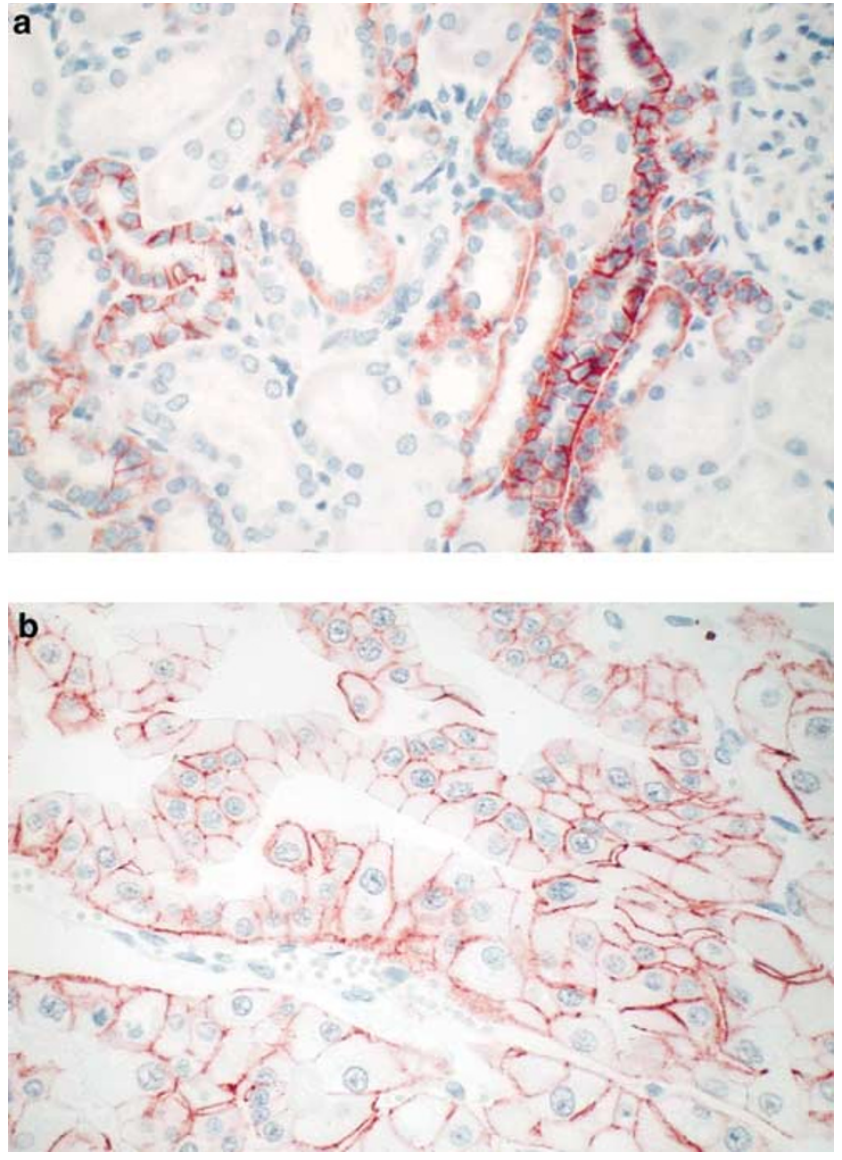

Figure 3 Strong membranous E-cadherin immunoreactivity of non-neoplastic distal tubule epithelium (a); note faint basal cytoplasmic staining of some proximal tubular cells. Distinct circumferential membranous staining of chromophobe renal cell carcinoma is shown in (b).

tively. In conventional carcinomas with sarcomatoid change, the sarcomatoid component always lacked E-cadherin immunoreactivity.

Differences with respect to cellular staining patterns for MUC1 were found between histological subtypes: conventional and papillary carcinomas presented with a predominantly membranous (either apical or circumferential, Figure 1b, c) or with mixed membranous and diffuse cytoplasmic staining (Figure 1d); no case with diffuse cytoplasmic staining without membranous immunoreacti vity was seen. Chromophobe carcinomas, in contrast, showed diffuse strong cytoplasmic staining (Figure 1f) in $17 / 22(77 \%)$ cases, a minority of the cases showing an additional membranous immunoreactivity $\left(P<0.0001 ; \chi^{2}\right.$ test $)$.

Furthermore, cellular staining patterns of MUC1positive conventional cancers showed a statistically significant association with both pT-stage $(P=0.002$; $\chi^{2}$ test) and tumor grade $\left(P=0.001 ; \chi^{2}\right.$ test): predominantly apical membranous staining was seen in low-stage (pT1/pT2 48/63 (76\%) vs pT3 20/49 (41\%); $P=0.0002$, Fisher's exact test) and low-grade $(\mathrm{G} 1 / \mathrm{G} 2 \quad 54 / 73 \quad(74 \%) \quad$ vs $\quad \mathrm{G} 3 / \mathrm{G} 4 \quad 14 / 39 \quad$ (36\%); 
$P=0.0001$, Fisher's exact test) tumors, whereas a predominantly circumferential membranous staining (with or without additional diffuse cytoplasmic immunoreactivity) prevailed in high-stage tumors. By comparing MA695 and E29 immunoreactivities, a significant agreement between the two antibodies was noted regarding different cellular staining patterns $\left(P<0.0001 ; \chi^{2}\right.$ test $)$.

\section{Survival Analysis}

A complete follow-up was available in 131/137 (96\%) patients with conventional renal cell carcinomas. After a mean follow-up of 26 months and a median follow-up of 24 months, progressive disease was observed in 29/131 (22\%) patients including 15 patients who died from cancer and 14 patients who are currently alive with metastatic disease. Three patients died from causes unrelated to renal cancer. The Cox proportional hazards regression model proved tumor stage and grade as independent prognostic factors regarding disease-free survival: stage pT3 tumors showed a relative risk (RR) of 4.1 (95\% confidence interval $=1.33-12.59, \quad P=0.01)$ compared to stages pT1 and pT2. The RR of grade 3 and 4 tumors was 17.2 (95\% confidence interval $=4.83-60.98, P<0.0001)$ compared to grades 1 and 2.

MUC1: Semiquantitative analysis of immunoreactivity yielded a significant influence on disease-free survival of patients with conventional tumors only in stages pT3a and pT3b (58 patients evaluable) using a cutoff value of $75 \%$ of positive cancer cells: 13/39 (33\%) patients with MUC1 expression in less than $75 \%$ of cancer cells (including MUC1-negative tumors) developed metastatic disease compared to 12/19 (63\%) patients with MUC1 immunoreactivity in more than $75 \%$ of cancer cells $(P=0.02$; log-rank test; Figure 4). With regard to analysis of cellular staining patterns (107 patients evaluable), however, highly significant differences in disease-free survival were detected: metastatic disease developed in

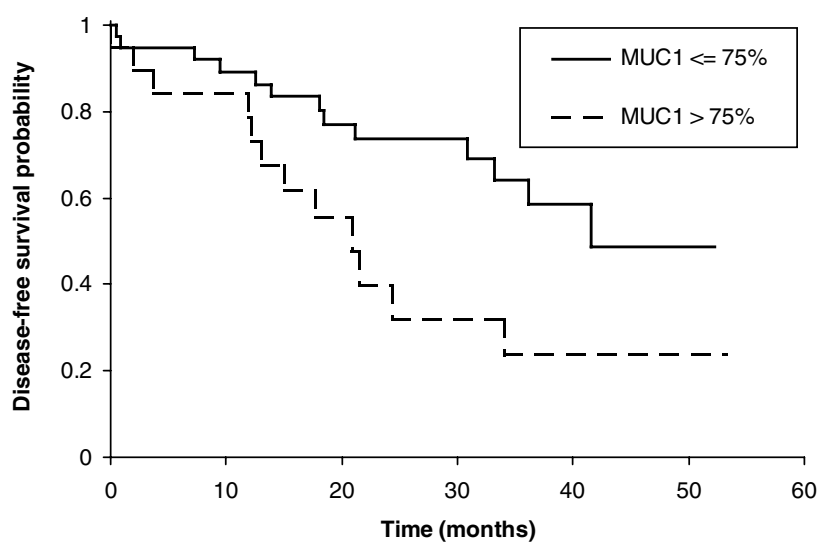

Figure 4 Disease-free survival (months) in patients with stage pT3 conventional renal cell carcinoma using a cutoff value of $75 \%$ for MUC1 immunoreactivity $(P=0.02$; log-rank test).

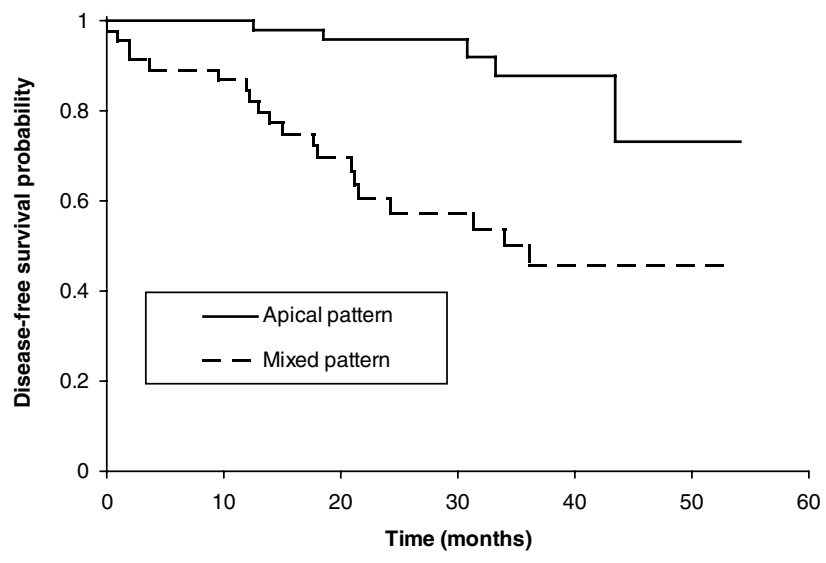

Figure 5 Disease-free survival (months) in patients with conventional renal cell carcinoma with predominantly apical membranous immunoreactivity compared to mixed patterns of predominantly circumferential membranous and additional diffuse cytoplasmic immunoreactivity for MUC1 $(P<0.0001$; logrank test).

5/61 (8\%) conventional tumors with predominantly apical membranous staining compared to $20 / 46$ $(43 \%)$ conventional tumors with predominantly circumferential membranous staining (with or without additional diffuse cytoplasmic staining; $P<0.0001$; log-rank test; Figure 5).

E-cadherin: No significant influence of E-cadherin expression on disease-free survival of patients with conventional renal cancers was noted: 26/124 (21\%) patients with E-cadherin-negative tumors compared to $3 / 7(43 \%)$ patients with E-cadherin-positive tumors developed metastatic disease. The difference was not significant $(P=0.3$; log-rank test).

\section{Discussion}

The immunoreactivity for MUC1 in the great majority of renal cell carcinomas in our series is in accordance with the results obtained in other studies, in which immunoreactivity with 'EMA'27,45-47 or 'MUC1'3-6,28 antibodies was observed in $43-100 \%$ of renal cell carcinomas. These studies mainly dealt with conventional cancers. However, by systematic analysis of the other histological subtypes (classified according to the consensus classification of renal neoplasia), we demonstrated that MUC1 immunoreactivity is valuable in differential diagnosis, especially when the cellular staining pattern is taken into account: the (predominantly) diffuse cytoplasmic staining was significantly associated with the chromophobe subtype. The few conventional renal cancers with diffuse cytoplasmic staining always showed a predominant circumferential membranous staining. For papillary carcinomas, our results confirmed the results by Leroy et $a l,{ }^{48}$ who noted a significantly higher MUC1 expression in type 1 compared to the more aggressive type 2 tumors, according to the work of Delahunt and Eble. ${ }^{49,50}$ Moreover, a 
systematic analysis comparing the antibody E29 (synonymous to EMA, raised against a delipidated extract of human milk fat and now known to be recognized as MUC1), with the novel antibody MA695 (raised against a carbohydrate epitope of the human MUC1 glycoprotein) has not yet been performed in renal cell carcinoma. Interestingly, the two different MUC1 antibodies showed similar, yet not identical results. A slightly weaker immuno reaction of E29 compared to MA695 was noted, which might be explained by differences in epitope masking due to different degrees of glycosylation. Regarding the E29 antibody, 35\% of conventional renal cell carcinomas lacked staining compared to $14 \%$ of chromophobe tumors. These results are compatible with other reports that noted EMA(E29) positivity in as much as $100 \%$ of chromophobe compared to $53-62 \%$ of conventional renal carcinomas. ${ }^{51,52}$ The MA695 antibody in our study, however, revealed similar semiquantitative staining results for both subtypes, supporting our opinion that for differentization of these types of cancer the analysis of staining patterns is essential.

MUC1 expression has been reported to be associated with high tumor grade and pT-stage as well as tumor progression in studies concentrating on the conventional subtype of renal cell carcinoma. ${ }^{3-6,27}$ These results are based on different criteria for evaluation of immunoreactivity, since some authors restricted themselves to a semiquantitative assessment, ${ }^{4,27}$ whereas others also considered staining patterns. ${ }^{3,5,6}$ With respect to semiquantitative analysis of MUC1 immunoreactivity, we were unable to reproduce the associations with pT-stage and grade. The stronger immunoreactivity of low-grade papillary carcinomas seems to be caused by stronger staining of the less aggressive type 1 papillary tumors. The additional analysis of staining patterns, however, revealed a statistically significant association with both pT-stage and tumor grade. Similar results were obtained in our survival analysis: by exclusive semiquantitative assessment of staining comparable to the work by Leroy et al, ${ }^{5,6}$ our results only reached low levels of significance. By considering the staining patterns, however, the results turned out to be highly significant, confirming the data obtained in breast cancer studies: ${ }^{26}$ While predominantly apical staining was associated with favorable outcome, the predominantly circumferential pattern was associated with poor prognosis, which was even worse, when an additional diffuse cytoplasmic staining was noted. Thus, the aberrant diffuse cytoplasmic localization of MUC1 seems to play an important role in determining disease outcome. It might reflect defects in processing and targeting pathways resulting from tumor cell dedifferentiation. ${ }^{26}$

According to the literature, E-cadherin expression can be found in 18-88\% of renal cell carcinomas. ${ }^{31-33,36}$ However, the validity of most studies is limited, since either the histological subtypes of renal cancer were not mentioned ${ }^{31,32}$ or were lumped together. $^{33,36}$ In our study, we found a significantly stronger reaction in chromophobe (95\%) compared to conventional renal cell carcinomas $(5 \%)$ the difference, however, was not as clear-cut as stated before by Taki et al, ${ }^{52}$ who reported $100 \%$ E-cadherin immunoreactivity (19/19) in chromophobe compared to complete lack of staining $(0 / 19)$ in conventional renal cancer cases. Interestingly, we noted a significant difference in staining between type 1 and type 2 papillary renal cell carcinomas, indicating that E-cadherin can be used as an additional marker for the separation of these two biologically distinct tumor entities. The lack of Ecadherin immunoreactivity in the sarcomatoid tumor components of 13 otherwise conventional tumors in our series confirms the report by Kuroiwa et $a l,{ }^{53}$ who showed a decrease in E-cadherin immunoreactivity in the sarcomatoid compared to the better differentiated components in their study of 11 cases of sarcomatoid renal cancers.

Whereas some authors noted a decrease of E-cadherin immunoreactivity with rising tumor grade and pT-stage, ${ }^{31,32,36}$ others failed to recognize this association and detected E-cadherin immuno reactivity even in the least differentiated tumors as well as in metastases. ${ }^{35}$ The stronger expression of E-cadherin in high-grade compared to low grade conventional cancers in our study suggests that impairment of E-cadherin function is independent of actual loss of the protein from tumor cells, as has been proposed earlier by Markovic-Lipkovski et al. ${ }^{33}$ Alternatively, E-cadherin expression may be of minor biological importance in renal cancer, since several other members of the cadherin family, especially N-cadherin and cadherin 6 , have been detected in larger quantities. Their diagnostic and prognostic significance, however, is still unclear. ${ }^{30,33,34,52,54}$ Finally, the stronger immunoreacti vity of high-grade papillary carcinomas is probably caused by the stronger staining of the more aggressive type 2 papillary tumors.

Regarding prognosis, E-cadherin expression was found to be independent of disease outcome in our series. The results of other authors reporting a significant association between loss of E-cadherin expression and tumor progression ${ }^{31,32}$ could not be reproduced. Since MUC1 is known to interfere with E-cadherin-mediated cell adhesion in vitro, ${ }^{11-13}$ we investigated a possible prognostic impact of MUC1 expression in patients with E-cadherin-positive renal cell carcinomas. As shown above, however, no influence could be detected in our series and, overall, no association between MUC1 and E-cadherin immunoreactivity was noted.

In summary, MUC1 immunoreactivity was shown to be of prognostic significance in conventional renal cell carcinomas, particularly when the cellular staining patterns were considered. Moreover, MUC1 and E-cadherin immunoreactivity proved to be valuable for the histopathological distinction of 
chromophobe and conventional as well as type 1 and type 2 papillary renal cancers. The two different MUC1 antibodies yielded comparable staining results. No association between MUC1 and E-cadherin immunoreactivity was found.

\section{Acknowledgements}

We are grateful to Mrs Gogg-Kammerer, Ms M Lindbauer, Ms A Sommersacher, Ms K Wagner, and $\mathrm{Mr} \mathrm{M}$ Al-Effah for excellent technical assistance.

\section{References}

1 Dekker J, Rossen JW, Buller HA, et al. The MUC family: an obituary. Trends Biochem Sci 2002;27:126-131.

2 Moniaux N, Escande F, Porchet N, et al. Structural organization and classification of the human mucin genes. Front Biosci 2001;6:1192-1206.

3 Fujita K, Denda K, Yamamoto M, et al. Expression of MUC1 mucins inversely correlated with post-surgical survival of renal cell carcinoma patients. Br J Cancer 1999;80:301-308.

4 Kraus S, Abel PD, Nachtmann C, et al. MUC1 mucin and trefoil factor 1 protein expression in renal cell carcinoma: correlation with prognosis. Hum Pathol 2002;33:60-67.

5 Leroy X, Copin MC, Devisme L, et al. Expression of human mucin genes in normal kidney and renal cell carcinoma. Histopathology 2002;40:450-457.

6 Leroy X, Zerimech F, Zini L, et al. MUC1 expression is correlated with nuclear grade and tumor progression in pT1 renal clear cell carcinoma. Am J Clin Pathol 2002;118:47-51.

7 Agrawal B, Krantz MJ, Parker J, et al. Expression of MUC1 mucin on activated human T cells: implications for a role of MUC1 in normal immune regulation. Cancer Res 1998;58:4079-4081.

8 Ligtenberg MJ, Buijs F, Vos HL, et al. Suppression of cellular aggregation by high levels of episialin. Cancer Res 1992;52:2318-2324.

9 Patton S, Gendler SJ, Spicer AP. The epithelial mucin, MUC1, of milk, mammary gland and other tissues. Biochim Biophys Acta 1995;1241:407-423.

10 Schroeder JA, Thompson MC, Gardner MM, et al. Transgenic MUC1 interacts with epidermal growth factor receptor and correlates with mitogen-activated protein kinase activation in the mouse mammary gland. J Biol Chem 2001;276:13057-13064.

$11 \mathrm{Li} \mathrm{Y,} \mathrm{Kuwahara} \mathrm{H,} \mathrm{Ren} \mathrm{J,} \mathrm{et} \mathrm{al.} \mathrm{The} \mathrm{c-Src} \mathrm{tyrosinase}$ kinase regulates signalling of the human DF3/MUC1 carcinoma-associated antigen with GSK3 beta and beta-catenin. J Biol Chem 2001;276:6061-6064.

12 Wesseling J, van der Valk SW, Hilkens J. A mechanism for inhibition of E-cadherin-mediated cell-cell adhesion by the membrane-associated mucin episialin/ MUC1. Mol Biol Cell 1996;7:565-577.

13 Yamamoto M, Bharti A, Li Y, et al. Interaction of the DF3/MUC1 breast carcinoma-associated antigen and beta-catenin in cell adhesion. J Biol Chem 1997;272: 12492-12494.
14 Hilkens J, Ligtenberg MJ, Vos HL, et al. Cell membraneassociated mucins and their adhesion-modulating property. Trends Biochem Sci 1992;17:359-363.

15 Baldus SE, Mönig SP, Hanisch FG, et al. Comparative evaluation of the prognostic value of MUC1, MUC2, sialyl-Lewis ${ }^{\mathrm{a}}$ and sialyl-Lewis ${ }^{\mathrm{x}}$ antigens in colorectal adenocarcinoma. Histopathology 2002;40:440-449.

16 Wittel UA, Goel A, Varshney GC, et al. Mucin antibodies - new diagnosis and therapy of cancer. Front Biosci 2001;6:1296-1310.

17 Kashiwagi H, Kijima H, Dowaki S, et al. MUC1 and MUC2 expression in human gallbladder carcinoma: a clinicopathological study and relationship with prognosis. Oncol Rep 2001;8:485-489.

18 Kimura T, Tanaka S, Haruma K, et al. Clinical significance of MUC1 and E-cadherin expression, cellular proliferation, and angiogenesis at the deepest invasive portion of colorectal cancer. Int J Oncol 2000;16:55-64.

19 Lee HS, Lee HK, Kim HS, et al. MUC1, MUC2, MUC5AC, and MUC6 expressions in gastric carcinomas: their roles as prognostic indicators. Cancer 2001;92:1427-1434.

20 Manne U, Weiss HL, Grizzle WE. Racial differences in the prognostic usefulness of MUC1 and MUC2 in colorectal adenocarcinomas. Clin Cancer Res 2000;6: 4017-4025.

21 Nakagawa K, Akagi J, Takai E, et al. Prognostic values of MUC-1 molecule expressing cytokine receptor-like epitope and DF3 in patients with gastric carcinoma. Int J Oncol 1999;14:425-435.

22 Utsonomiya $\mathrm{T}$, Yonezawa $\mathrm{S}$, Sakamoto $\mathrm{H}$, et al. Expression of MUC1 and MUC2 mucins in gastric carcinomas: its relationship with the prognosis of the patients. Clin Cancer Res 1998;4:2605-2614.

23 Sagara M, Yonezawa S, Nagata T, et al. Expression of mucin 1 (MUC1) in esophageal squamous-cell carcinoma: its relationship with prognosis. Int J Cancer 1999;84:251-257.

24 Sivridis E, Giatromanolaki A, Koukourakis MI, et al. Patterns of episalin/MUC1 expression in endometrial carcinomas and prognostic relevance. Histopathology 2002;40:92-100.

25 Guddo F, Giatromanolaki A, Koukourakis MI, et al. MUC1 (episalin) expression in non-small cell lung cancer is independent of EGFR and c-erbB-2 expression and correlates with poor survival in node positive patients. J Clin Pathol 1998;51:667-671.

26 Rahn JJ, Dabbagh L, Pasdar M, et al. The importance of MUC1 cellular localization in patients with breast carcinoma: an immunohistologic study of 71 patients and review of the literature. Cancer 2001;91: 1973-1982.

27 Bamias A, Chorti M, Deliveliotis C, et al. Prognostic significance of CA 125, CD44, and epithelial membrane antigen in renal cell carcinoma. Urology 2003;62:368-373.

28 Cao Y, Karsten U, Zerban H, et al. Expression of MUC1, Thomsen-Friedenreich-related antigens, and cytokeratin 19 in human renal cell carcinomas and tubular clear cell lesions. Virchows Arch 2000;436:119-126.

29 Denda-Nagai K, Fujita K, Fujime M, et al. Absence of correlation of MUC1 expression to malignant behavior of renal cell carcinoma in experimental systems. Clin Exp Metast 2000;18:77-81.

30 Heicappel R. Cadherins in renal cell carcinoma. Anticancer Res 1999;19:1501-1504. 
31 Fischer C, Georg CH, Kraus S, et al. CD44s, E-cadherin and PCNA as markers of progression in renal cell carcinoma. Anticancer Res 1999;19:1513-1518.

32 Katagiri A, Watanabe R, Tomita Y. E-cadherin expression in renal cell cancer and its significance in metastasis and survival. Br J Cancer 1995;71:376-379.

33 Markovic-Lipkovski J, Brasanac D, Muller GA, et al. Cadherins and integrins in renal cell carcinoma: an immunohistochemical study. Tumori 2001;87:173178.

34 Shimazui T, Giroldi LA, Bringuier PP, et al. Complex cadherin expression in renal cell carcinoma. Cancer Res 1996;56:3234-3237.

35 Tani T, Laitinen L, Kangas L, et al. Expression of E- and $\mathrm{N}$-cadherin in renal cell carcinomas, in renal cell carcinoma cell lines in vitro and in their xenografts. Int J Cancer 1995;64:407-414.

36 Terpe HJ, Tajrobekhar K, Günthert U, et al. Expression of cell adhesion molecules alpha-2, alpha-5 and alpha6, integrin, E-cadherin, N-CAM and CD44 in renal cell carcinomas. An immunohistochemical study. Virchows Arch 1993;422:219-224.

37 Sobin LH, Wittekind C, (eds). TNM Classification of Malignant Tumors. 6th edn. Wiley-Liss Inc: New York, 2002, pp 181-183.

38 Fuhrman SA, Lasky LC, Limas C. Prognostic significance of morphologic parameters in renal cell carcinoma. Am J Surg Pathol 1982;6:655-663.

39 Kovacs G, Akhtar M, Beckwith BJ, et al. The Heidelberg classification of renal cell tumours. J Pathol 1997;183:131-133.

40 Störkel S, Eble JN, Adlakha K, et al. Classification of renal cell carcinoma: Workgroup No 1. Union Internationale Contre le Cancer (UICC) and the American Joint Committee on Cancer (AJCC). Cancer 1997; 80:987-989.

41 Kononen J, Bubendorf L, Kallioniemi A, et al. Tissue microarrays for high-throughput molecular profiling of tumor specimens. Nat Med 1998;4:844-847.

42 Milanes-Yearsley M, Hammond MEH, Pajak TF, et al. Tissue micro-array: a cost and time-effective method for correlative studies by regional and national cancer study groups. Mod Pathol 2002;15:1366-1373.

$43 \mathrm{Hsu}$ FD, Nielsen TO, Alkushi A, et al. Tissue microarrays are an effective quality assurance tool for diagnostic immunohistochemistry. Mod Pathol 2002; 15:1374-1380.

44 Cao Y, Karsten U, Hilgers J. Immunohistochemical characterization of a panel of 56 antibodies with normal human small intestine, colon, and breast tissues. Tumour Biol 1998;19(Suppl 1):88-99.

45 Fleming S, Lindop GB, Gibson AA. The distribution of epithelial membrane antigen in the kidney and its tumours. Histopathology 1985;9:729-739.

46 Medeiros LJ, Michie LJ, Johnson DE, et al. An immunoperoxidase study of renal cell carcinomas: correlation with nuclear grade, cell type, and histo logic pattern. Hum Pathol 1988;19:980-987.

47 Banner BF, Burnham JA, Bahnson RR, et al. Immunophenotyic markers in renal cell carcinoma. Mod Pathol 1990;3:129-134.

48 Leroy X, Zini L, Leteurtre E, et al. Morphologic subtyping of papillary renal cell carcinoma: correlation with prognosis and differential expression of MUC1 between the two subtypes. Mod Pathol 2002;15: 1126-1130.

49 Delahunt B, Eble JN. Papillary renal cell carcinoma: a clinicopathologic and immunohistochemical study of 105 tumors. Mod Pathol 1997;10:537-544.

50 Delahunt B, Eble JN, McCredie MRE, et al. Morpho logic typing of papillary renal cell carcinoma: comparison of growth kinetics and patients survival in 66 cases. Hum Pathol 2001;32:590-595.

51 Khoury JD, Abrahams NA, Levin HS, et al. The utility of epithelial membrane antigen and vimentin in the diagnosis of chromophobe renal cell carcinoma. Ann Diagn Pathol 2002;6:154-158.

52 Taki A, Nakatani Y, Misugi K, et al. Chromophobe renal cell carcinoma: an immunohistochemical study of 21 Japanese cases. Mod Pathol 1999;12:310-317.

53 Kuroiwa K, Konomoto T, Kumazawa J, et al. Cell proliferation activity and expression of cell-cell adhesion factors (E-cadherin, apha-, beta-, and gamma-catenin, and p120) in sarcomatoid renal cell carcinoma. J Surg Oncol 2001;77:123-131.

54 Paul R, Ewing CM, Robinson JC, et al. Cadherin-6, a cell adhesion molecule specifically expressed in the proximal renal tubule and renal cell carcinoma. Cancer Res 1997;57:2741-2748. 